\title{
A Novel Technique for Bladder Wall Fixation in Pneumovesicoscopic Surgery: T-Bar
}

\author{
Beytullah Yağız, MD, ${ }^{1,2}$ and Özlem Balcı, MD ${ }^{1}$
}

\begin{abstract}
Background: As a relatively new approach, popularity of pneumovesicoscopic surgery is increasing, but slower than expected due to complex nature of the procedure with efforts to overcome the difficult steps of the procedure. Bladder fixation is one of the crucial steps of the procedure. In this study, we present a novel and simple T-bar technique to overcome this difficulty.

Methods: We retrospectively evaluated 24 consecutive patients (39 ureters) who underwent pneumovesicoscopic surgery with fixation of the bladder wall between December 2017 and September 2019.

Results: Fixation by transabdominal suture (TS) was performed in 3 patients, while fixation by thread loops with needle in 3 and T-bar device in 18. Tearing of the bladder wall was encountered in 2 patients in TS, in 2 patients with thread loop groups, but none in the T-bar group. Conversion to open surgery was necessary in 3 patients in the T-bar group, but only 1 was related with the fixation technique.

Conclusions: T-bar technique is an inexpensive and simple solution providing stable and reliable bladder wall and working port fixation during pneumovesicoscopy.
\end{abstract}

Keywords: pneumovesicoscopy, bladder, fixation, T-bar

\section{Introduction}

$\mathbf{I}^{\mathrm{r}}$ NTRAVESICAL SURGICAL PROCEDURES by means of pneumovesicoscopy is gaining popularity, but popularity and acceptance seem slower than expected. Increasing bulk of evidence on the pneumovesicoscopic surgery demonstrates similar succes rates with the open techniques. ${ }^{1-4}$

One of the crucial steps of pneumovesicoscopy is fixing the bladder wall to the abdominal wall as failure of this step may result in complications, termination of pneumovesicoscopy, and conversion to open surgery. ${ }^{5}$ Some special devices such as locking trocars, ports with umbrella, and single-use lifting equipments have been commercially introduced for bladder fixation. ${ }^{2,5}$ Although they are very useful and practical, they have not gained widespread acceptance. Significant cost of these devices prevent their widespread use as many other simple techniques without using a special device or equipment are described to reduce the cost of the bladder fixation during pneumovesicoscopy. 6,7

In this study, we reviewed our experience with some of these fixation techniques and present our simple technique performed by means of a homemade device: T-bar.

\section{Materials and Methods}

Twenty-four consecutive patients (39 ureters) who underwent pneumovesicoscopic surgery for various conditions with fixation of the bladder wall between December 2017 and September 2019 are included in the study. Sixteen of the patients were girls and 8 were boys. Fifteen patients were bilateral and 9 were unilateral. The pitfalls and complications are retrospectively evaluated. An Institutional Review Board Approval is obtained (IRBA no: 2019/12-12). All operative procedures were performed by a single surgeon.

\footnotetext{
${ }^{1}$ Department of Pediatric Surgery, Dr Sami Ulus Gynecology, Obstetrics and Child Health and Diseases Training and Research Hospital, Ankara, Turkey.

${ }^{2}$ Department of Pediatric Urology, Dr Sami Ulus Gynecology, Obstetrics and Child Health and Diseases Training and Research Hospital, Ankara, Turkey.

A video demonstrating this technique is available online.
} 


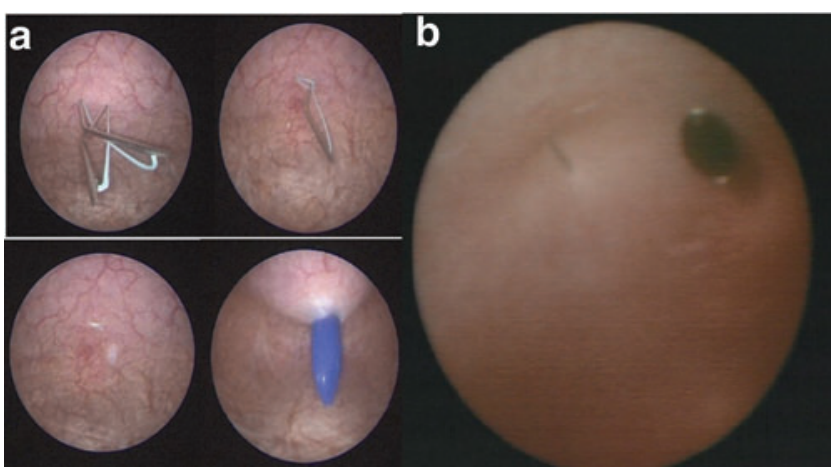

FIG. 1. Bladder wall fixation by NAPF (a) and TS (b) techniques. A large needle is traversing the abdominal and bladder wall on the left of the image (b). NAPF, needleassisted percutaneous fixation; TS, transabdominal suture.

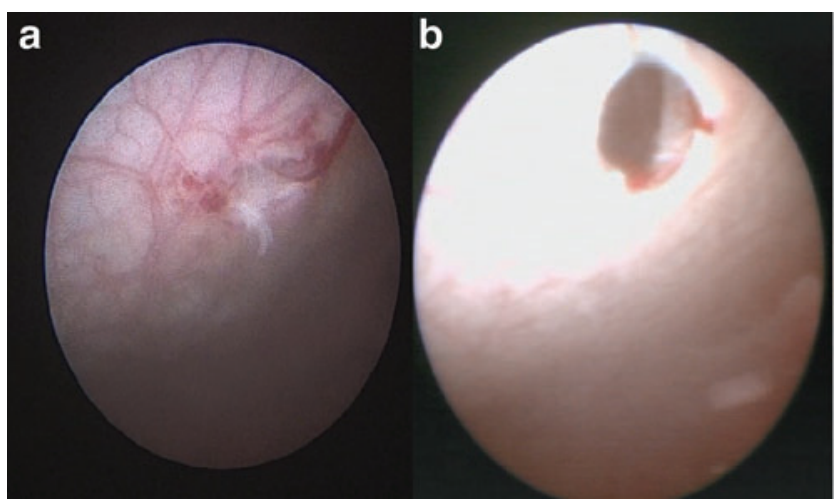

FIG. 2. Bladder tear encountered with NAPF (a) and TS (b) techniques.

\section{Operative Technique}

The procedure starts with cystoscopy and the patient is placed in lithotomy position. In the needle-assisted percutaneous fixation (NAPF) technique, bladder wall is fixed percutaneously by the help of thread loops loaded on injection needles under cystoscopic guidance, similar to the principle of percutaneous internal ring suturing method described by
Patkowski et al. (Fig. 1a). ${ }^{8}$ In the transabdominal suture (TS) technique, the bladder wall was fixed by passing a large needle through the abdominal and bladder wall under cystoscopic vision (Fig. 1b). Tearing of the bladder wall was encountered in both techniques (Fig. 2a, b).

In the T-bar technique, a $2-3 \mathrm{~cm}$ fragment is cut from a $5 \mathrm{~F}$ polyurethane stiff ureteral catheteter and a $2 / 0$ suture is passed and returned $0.5 \mathrm{~cm}$ away in the middle part of the catheter forming a figure of "T" (Fig. 3a). This apparatus is prepared for each of the working ports. T-bar apparatuses are introduced in to the bladder over a guide wire by the aid of cystoscopy. A suture retrieval loop is prepared with a monofilament suture introduced through a 20G injection needle, as in the PIRS technique (Fig. 3b). ${ }^{8}$ Under cystoscopic vision, the needle of retrieval loop is introduced in to the bladder through the abdominal wall from the selected site, the thread of T-bar is grasped by the retrieval loop inside the bladder with the help of a forceps under cystoscopic guidance and then thread is taken out anchoring the bladder wall to the abdominal wall by the T-bar (Fig. 4).

This step is repeated for all of the port sites and then ports are introduced in to the bladder under cystoscopic vision (Fig. 5). Although some may prefer carbon dioxide insufflation during cystoscopic stage, we preferred distending the bladder with fluid because fluid is less compressible than gas and provides safer and easier port insertion. ${ }^{4}$ Ports are fixed by free ends of the T-bar threads outside the abdominal wall (Fig. 5). Afterward, cystoscopic step of the procedure is finished and patient is repositioned for pneumovesicoscopic step of the procedure. After the entire procedure is over, threads are released and T-bars are dropped in the bladder and taken out under direct vision through the working ports (See Supplementary Video S1 for details).

\section{Results}

Sixteen of the patients were girls and 8 were boys. Median age at operation was 5.5 years (range $1.5-14.8$ years). The indications for surgery was vesicoureteral reflux (VUR) in 16 , isolated bladder diverticulum in 3, bladder diverticulum with VUR in 2, obstructing and refluxing megaureter in 2 , and primary obstructing megaureter in 1 .

Under cystoscopic guidance, bladder wall was secured to the abdominal wall by different techniques in the first 6 patients and by T-bar technique in the last 18 . Bladder wall was

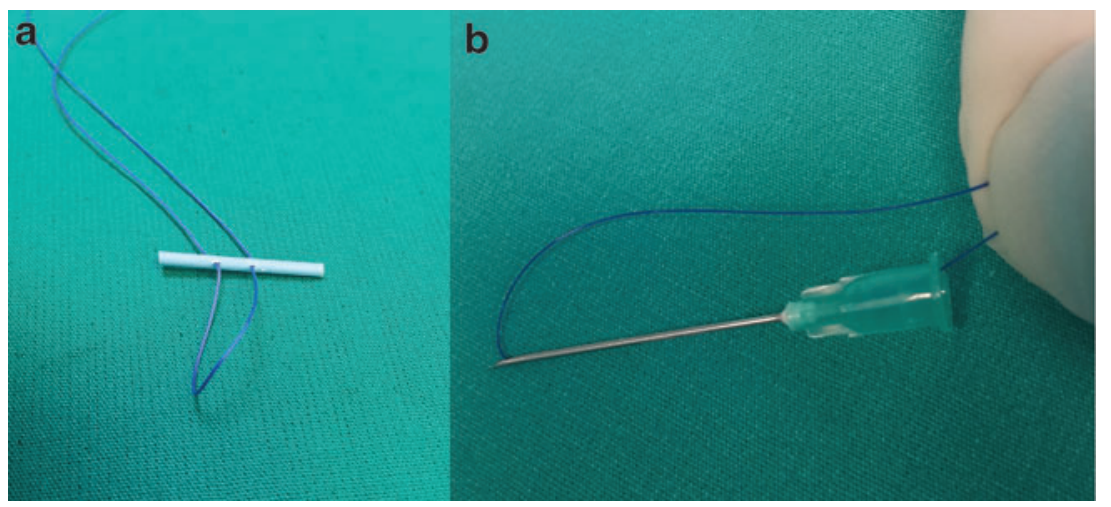

FIG. 3. T-bar (a) and retrieval loop (b) are prepared at the operating table with a polyurethane ureteral catheter fragment, a $20 \mathrm{G}$ needle and threads. 


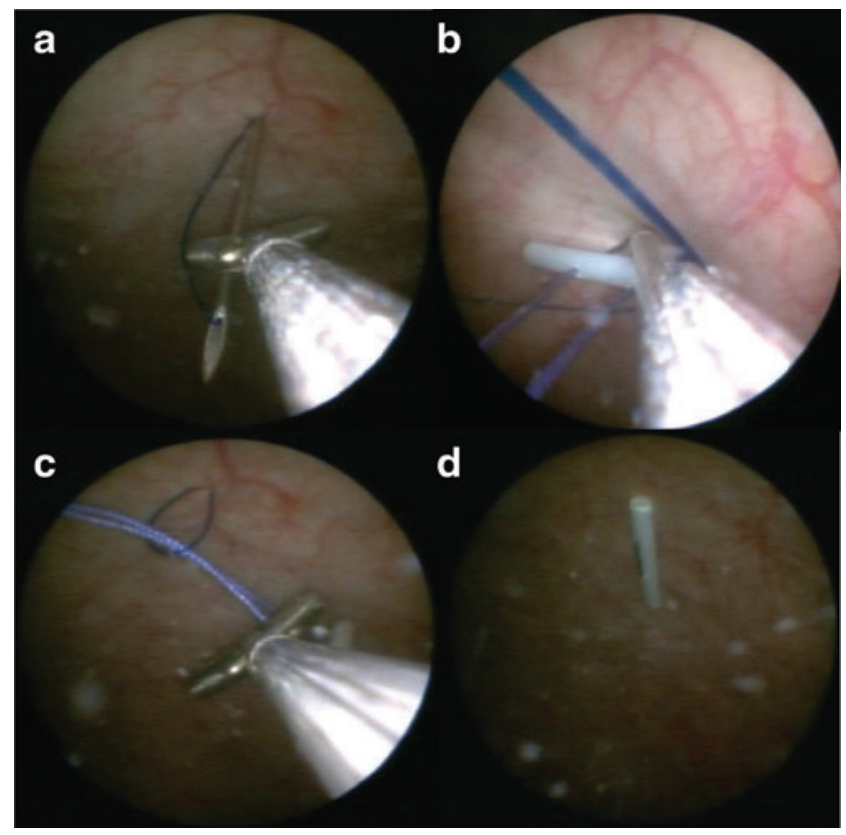

FIG. 4. Under cystoscopic guidance, needle of the retrieval loop is punctured through the abdominal wall (a), T-bar is caught by the loop with the help of a forceps (b), the loop is taken out retrieving the free ends of thread (c) and T-bar stabilizes the bladder wall (d). fixed in 3 patients by TS technique and by NAPF technique in 3. Bladder wall was torn by the needle or the thread in 2 and 2 patients in the TS and NAPF groups, respectively. Although no conversion was required in any of these patients, maintaining the bladder wall and port fixation were troublesome and required additional maneuvers that prolonged the surgical duration. No bladder wall tearing was encountered in the T-bar group.

Conversion to open surgery was required in 3 patients in the T-bar group, but only 1 was related with the bladder wall fixation technique. One conversion was due to massive intraperitoneal air leak that prevented maintaining pneumovesicoscopy and the other one was secondary to failure of safe ureteral dissection due to fibrotic neurogenic bladder with thick wall. Conversion to open was due to injury of the epigastric artery in the third patient during abdominal puncture with the needle for retrieval of the T-bar. The bleeding could not be controlled and conversion to open was needed due to poor vision, and epigastric artery was ligated to stop bleeding.

Port dislodgement is encountered in 1,1 , and 1 patients in the TS, NAPF, and T-bar groups, respectively. Port dislodgement was easily managed in T-bar group, thanks to the T-bar. In the other groups, ports were dislocated because the fixation threads tore all the layers of the bladder during pneumovesicoscopic stage of the procedure and bladder wall was needed to be fixed again before reinsertion of the ports, which is a more complicated and troublesome manuever at this stage of the procedure. No bladder wall drop or tear is encountered in the T-bar group.
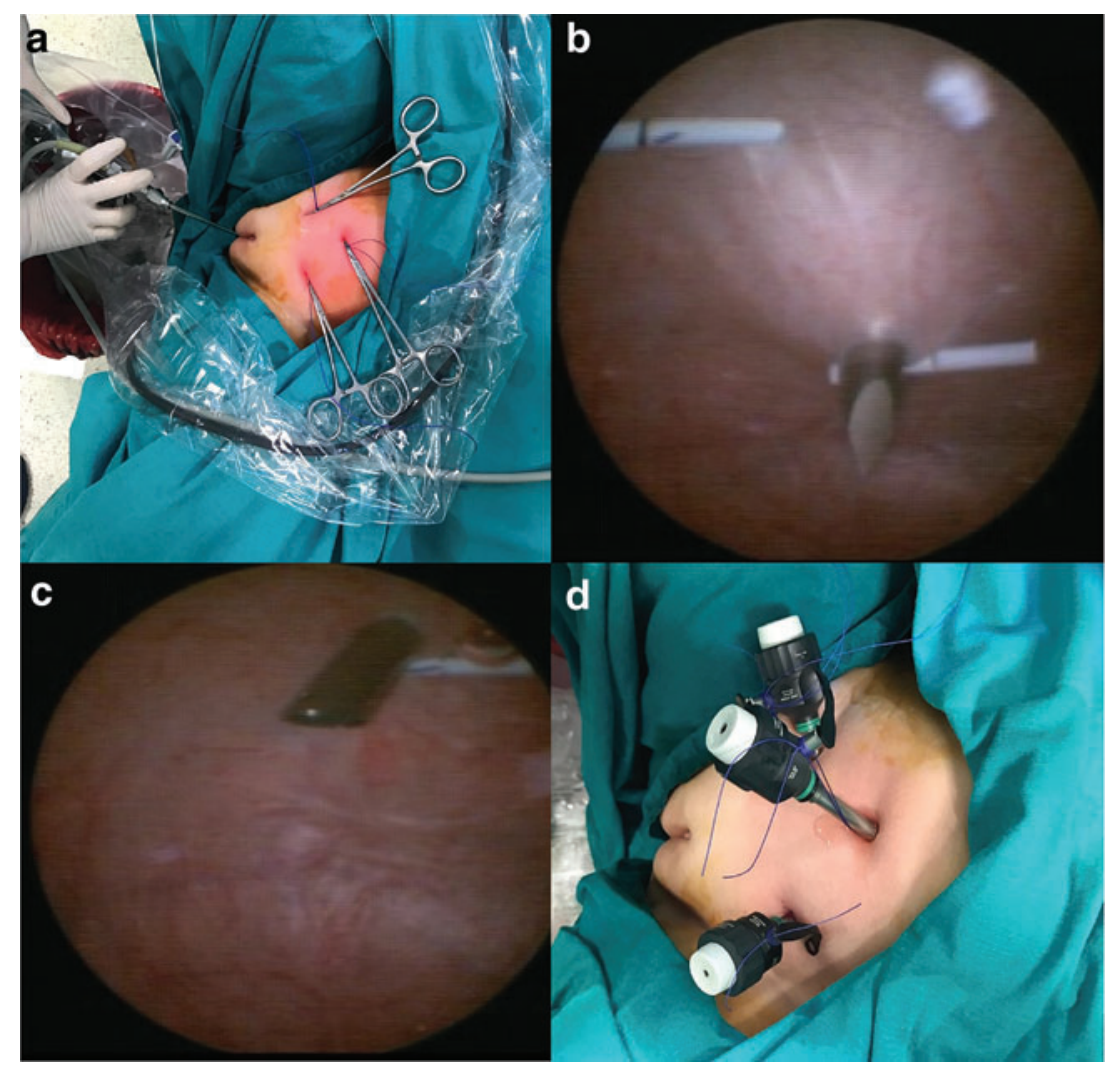

FIG. 5. Free ends of the T-bar are tightened outside the abdominal wall (a) and working ports are inserted with the bladder distended with fluid (b and $\mathbf{c}$ ), and fixed by the threads outside (d). 
As a separate step, median cytoscopy time was 26 minutes (range, 22-36 minutes) in the T-Bar group and 29 minutes (range, 23-35) in the rest of the patients.

\section{Discussion}

Although popularity of minimal invasive surgery is increasing, pneumovesicoscopic surgical procedures have not gained acceptance that much. Some of the reasons are complex nature of these procedures, requirement of advanced laparoscopic skill, longer procedural duration than conventional techniques, and limited data on the success rates. Bladder wall fixation is one of the crucial steps of the procedure and extends an already long operation with further contribution to the complexity of the procedure and discourages the clinician. There are many specific devices to shorten and facilitate this step, but have not reached widespread use and are not widely available due to commercial reasons and their cost.

Yeung et al. described suspending the bladder by a TS and reported 3/16 (19\%) air leak with $1(6 \%)$ conversion to open surgery, but stated that all were before the use of suspension suture. ${ }^{7}$ Although the technique is simple and useful, it is hard to perform in children with thick abdominal wall and tearing of the bladder wall was a significant problem in our experience. Some others reported suspending the bladder by simple needle and thread loops, a principle described by Patkowski et al. for percutaneous laparoscopic inguinal hernia repair, but we experienced bladder wall tearing with this technique too. ${ }^{8,9}$ Lau et al. reported bladder suspension with a T-shaped commercially available single-use equipment without any complication related to it, but the cost and availability of the device are the factors restricting its use. ${ }^{5}$

Home made T-bar system is inexpensive, simple, and reliable. The ureteral catheter at the end of the T-bar prevents bladder tearing by distributing the traction force on the wider surface of the bar, provides more stable fixation for bladder wall and for the working ports. The only complication we experienced with the T-bar was injury to the epigastric artery during puncture of the abdominal wall with needle. This can be considered as a general complication of open or minimally invasive abdominal surgery, in which abdominal wall is needed to be traversed surgically. Additional cystoscopy time needed for bladder fixation is reasonable, and preparation of the cystoscopy table and positioning and repositioning of the patient are the main time-consuming parts of this step of the procedure.

\section{Conclusion}

Bladder wall fixation is a crucial step of pneumovesicoscopic surgery and T-bar technique is a safe and reliable method for bladder wall fixation during pneumovesicoscopy as a simple and inexpensive solution. We hope that further experience by others and us will provide more data about the reliability, pitfalls, and complications of our technique.

\section{Disclosure Statement}

No competing financial interests exist.

\section{Funding Information}

No grant or funding was received for this study.

\section{Supplementary Material}

Supplementary Video S1

\section{References}

1. Emir H, Mammadov E, Elicevik M, Buyukunal C, Soylet Y. Transvesicoscopic cross-trigonal ureteroneocystostomy in children: A single-center experience. J Pediatr Urol 2012;8: 83-86.

2. Valla JS, Steyaert H, Griffin SJ, Lauron J, Fragoso AC, Arnaud P, et al. Transvesicoscopic Cohen ureteric reimplantation for vesicoureteral reflux in children: A singlecentre 5-year experience. J Pediatr Urol 2009;5:466-471.

3. Chung KLY, Sihoe J, Liu K, Chao N, Hung J, Liu C, et al. Surgical outcome analysis of pneumovesicoscopic ureteral reimplantation and endoscopic dextranomer/hyaluronic acid injection for primary vesicoureteral reflux in children: A multicenter 12-year review. J Laparoendosc Adv Surg Tech A 2018;28:348-353.

4. Badawy H, Eid A, Hassouna M, Elkarim AA, Elsalmy S. Pneumovesicoscopic diverticulectomy in children and adolescents: Is open surgery still indicated? J Pediatr Urol 2008;4:146-149.

5. Lau CT, Lan LCL, Wong KKY, Tam P. Pneumovesical ureteric reimplantation using T-fastener: A modification for bladder wall anchorage. J Pediatr Urol 2017;13:326-328.

6. Soh S, Kobori Y, Shin T, Suzuki K, Iwahata T, Sadaoka Y, et al. Transvesicoscopic ureteral reimplantation: Politano-Leadbetter versus Cohen technique. Int J Urol 2015;22:394-399.

7. Yeung CK, Sihoe JD, Borzi PA. Endoscopic cross-trigonal ureteral reimplantation under carbon dioxide bladder insufflation: A novel technique. J Endourol 2005;19:295-299.

8. Patkowski D, Czernik J, Chrzan R, Jaworski W, Apoznanski W. Percutaneous internal ring suturing: A simple minimally invasive technique for inguinal hernia repair in children. J Laparoendosc Adv Surg Tech A 2006;16:513-517.

9. Jayanthi VR. Vesicoscopic cross-trigonal ureteral reimplantation: High success rate for elimination of primary reflux. J Pediatr Urol 2018;14:324.e1-.e5.

Address correspondence to: Beytullah Yă̆ız, MD

Department of Pediatric Surgery

Dr Sami Ulus Gynecology, Obstetrics and Child Health and Diseases Training and Research Hospital

Beştepeler Mah, Alparslan Türkeş Cad. No: 27 Beştepe

Ankara 06560

Turkey

E-mail: beytullahyagiz@gmail.com 